\title{
Design of Nano- and Microfiber Combined Scaffolds by Electrospinning of Collagen onto Starch-Based Fiber Meshes: A Man-Made Equivalent of Natural Extracellular Matrix
}

\author{
Kadriye Tuzlakoglu, B.Eng., M.Sc., Ph.D., ${ }^{1,2, *}$ Marina I. Santos, B.Sc., Ph.D., ${ }^{1,2, *}$ \\ Nuno Neves, B.Eng., M.Sc., Ph.D., ${ }^{1,2}$ and Rui L. Reis, B.Eng., M.Sc., Ph.D.,
}

Mimicking the structural organization and biologic function of natural extracellular matrix has been one of the main goals of tissue engineering. Nevertheless, the majority of scaffolding materials for bone regeneration highlights biochemical functionality in detriment of mechanical properties. In this work we present a rather innovative construct that combines in the same structure electrospun type I collagen nanofibers with starchbased microfibers. These combined structures were obtained by a two-step methodology and structurally consist in a type I collagen nano-network incorporated on a macro starch-based support. The morphology of the developed structures was assessed by several microscopy techniques and the collagenous nature of the nanonetwork was confirmed by immunohistochemistry. In addition, and especially regarding the requirements of large bone defects, we also successfully introduced the concept of layer by layer, as a way to produce thicker structures. In an attempt to recreate bone microenvironment, the design and biochemical composition of the combined structures also envisioned bone-forming cells and endothelial cells (ECs). The inclusion of a type I collagen nano-network induced a stretched morphology and improved the metabolic activity of osteoblasts. Regarding ECs, the presence of type I collagen on the combined structures provided adhesive support and obviated the need of precoating with fibronectin. It was also importantly observed that ECs on the nano-network organized into circular structures, a three-dimensional arrangement distinct from that observed for osteoblasts and resembling the microcappillary-like organizations formed during angiogenesis. By providing simultaneously physical and chemical cues for cells, the herein-proposed combined structures hold a great potential in bone regeneration as a man-made equivalent of extracellular matrix.

\section{Introduction}

I N BONE TISSUE ENGINEERING, the material selection lies at the very heart of the scaffold design. Up to date, various alternatives, such as metals, ceramics, and polymers, have been proposed to be used as scaffold materials. However, nowadays scaffolds are typically fashioned from biodegradable materials of natural origin proteins like collagen ${ }^{1}$ and silk fibroin, ${ }^{2}$ and polymers like chitosan, ${ }^{3}$ starch, ${ }^{4}$ and poly(3-hydroxybutyrate), ${ }^{5}$ and also from synthetic polymers such as poly(lactide), ${ }^{6}$ poly(lactide/glycolide), ${ }^{7}$ and polycaprolactone. ${ }^{8}$ Although synthetic polymers appear to be a good choice regarding processing, critical problems in biocompatibility, degradation products, and numerous other issues still remain to be solved. Conversely, naturally derived materials offer some advantages in terms of biocompatibility, as well as biochemical functionality by showing similarity to structures in animal tissues. The use of collagen as a scaffold is distinct from other polymers mainly due to its role in the formation of tissue and organs. It is the most abundant mammalian protein accounting for about $20 \%$ $30 \%$ of total body proteins. ${ }^{9}$ Collagen assembles into different supramolecular structures in natural extracellular matrix (ECM) of tissues and has exceptional functional diversity.

ECM is a complex composite of various proteins in fibrillar form and glycosaminoglycans chains ${ }^{10}$ and provides an important model for scaffold design. This network structure serves as a scaffold that can support tensile and compressive stresses by the fibrils and hydrated networks. Besides providing an appropriate microenvironment for cells, ECM is responsible for transmitting signals to cell membrane receptors that reach nucleus via intracellular signaling cascades. Therefore, the fibrillar and porous

\footnotetext{
${ }^{1} 3 B^{\prime}$ s Research Group-Biomaterials, Biodegradables and Biomimetics, Headquarters of the European Institute of Excellence on Tissue Engineering and Regenerative Medicine, University of Minho, Guimarães, Portugal.

${ }^{2}$ PT Associated Laboratory, IBB-Institute for Biotechnology and Bioengineering, Guimarães, Portugal.

*These two authors contributed equally to this work.
} 
structure of ECM has a great influence on cell functionality, mainly on cell adhesion and migration.

The development of suitable scaffolds, man-made systems that can mimic the structural organization and biologic function of natural ECM, remains a major aim for tissue engineers. In recent years, the electrospinning processes have received substantial attention as a way to mimic the structure of natural ECM by means of producing fibers down to $3 \mathrm{~nm}{ }^{11}$ This is due to the architectural similarity of the nonwoven mats, composed of electrospun nanofibers, to collagen structure of ECM. However, the pore sizes of electrospun mats, which are smaller than a cellular diameter, cannot allow for cell migration within the structure and results in a scaffold surface covered by a film of cells. Such type of systems cannot be used for tissue engineering of three-dimensional (3D) tissues. Further, the small size of the fibers tends not to maximize the points of cell attachment, which is a negative effect on expression of several factors and on cell spreading and differentiation.

When engineering bone, the scaffold must meet the mechanical properties of the tissue while it should also ideally mimic the biological task of ECM. Bone tissue is composed of a heterogeneous mixture of cell types embedded within a mineralized ECM. ${ }^{12}$ To assure the requirements of this metabolic active tissue, bone microenvironment is supplied by a complex intraosseous circulation composed by an intricate network of arteries, capillaries, and veins. ${ }^{13}$ Type I collagen is the major organic component of the osseous ECM. Besides its structural role, this ECM protein also promotes cell adhesion in an integrin-mediated fashion. ${ }^{14}$ In addition, type I collagen modulates cell-specific functions. In the osteogenic lineage it promotes osteogenic differentiation, proliferation, and mineralization. $^{12,15}$ Endothelial cells (ECs) are pivotal cells in blood vessel formation, and it is known that interstitial type I collagen induces the directional migration and lumen formation during angiogenesis. ${ }^{16,17}$ In fact osteogenesis and angiogenesis are two phenomena that cannot be dissociated during skeletal development, fracture repair, as well as in bone tissue engineering. ${ }^{18}$ It is well known that prompt revascularization favors osteoblastic differentiation, whereas prolonged hypoxia favors formation of cartilage or fibrous tissue. ${ }^{19}$ In bone tissue engineering, vascularization is necessary not only for new bone formation but also for the survival of the implanted cells on the carrier material after implantation. ${ }^{20}$ Accordingly, strategies that enhance angiogenesis should have positive effects on bone repair. ${ }^{21}$

Most approaches to engineering new tissue relied on the host for vascularization, but this is clearly not successful in thick and highly vascularized tissues such as bone. ${ }^{22}$ Hence, the need for proper vascularization, which involves the creation of a microvascular network and a macroscopic circulation, remains one of the major problems for larger tissue-engineered structures. ${ }^{23}$ To create a vascularized scaffold, a number of methods have been proposed. ${ }^{24,25}$ One approach involves the transplantation of ECs in an effort to engineer a vascular network from these cells, rather than waiting for host-blood-vessel ingrowth. ${ }^{26}$ However, independently of the adopted approach to accelerate vascularization, all of them will involve directly or indirectly ECs. Therefore, the key success for vascularized bone is the development of a structure that includes not only boneforming cells but also ECs.
We herein propose for the first time the use of combined structures as a man-made equivalent of natural ECM for bone tissue engineering. These constructs combine a macro support, microfiber meshes made from a blend of starch with polycaprolactone (SPCL), with a nano-network of electrospun type I collagen. These structures were designed envisioning formation of a mineralized matrix supplied by a vascular network. Therefore, in this work we have characterized the developed structures from the chemical and structural point of view, and assessed the cellular responses of bone-forming cells and ECs.

\section{Materials and Methods}

\section{Materials}

The material used in the production of microfiber meshes was a SPCL blend $(30 / 70 \mathrm{w} / \mathrm{w})$. More details on this material can be found elsewhere. . $^{47}$

Collagen was isolated from Wistar rat tails according to a typical acid extraction procedure. ${ }^{28}$ Briefly, the rat tails from sacrificed animals were cut off and soaked in ethanol (70\%) for $1 \mathrm{~min}$. The tendons were then pulled out and dissolved in sterile acetic acid $(0.5 \mathrm{M})$. The resulted solution was filtered through a sterile muslin gauze and freeze-dried. All the reagents used were analytical grade unless specified otherwise.

\section{Production of nano- and microfiber combined structures}

Wet spinning. Starch-based fiber meshes were fabricated by wet spinning methodology as described elsewhere. ${ }^{4}$ In a typical procedure, a viscous polymer solution was obtained by dissolving SPCL in chloroform $(40 \% \mathrm{w} / \mathrm{v})$. Methanol was used as a coagulant. A syringe pump (World Precision Instruments) was used to extrude a certain amount of polymer into a coagulation bath. The fiber mesh structure was formed during the processing by moving of the coagulation bath randomly. The fiber meshes were then dried at room temperature (RT) overnight to remove remaining solvents.

Electrospinning. To obtain collagen nanofibers on the wet-spun SPCL fiber meshes, an electrospinning method was used. Collagen $(0.85 \mathrm{mg})$ was dissolved in $1 \mathrm{~mL}$ of $1,1,1,3,3,3-$ hexafluoro-2-propanol and thoroughly mixed until the dissolution completed. The polymer solution was put into a syringe and placed in a syringe pump. A positive highvoltage supplier was used to maintain the voltage at $20 \mathrm{kV}$. The voltage was applied between the syringe tip and a ground plate, where the fiber mesh membranes were placed, during $10 \mathrm{~s}$. Both sides of the membranes were impregnated with collagen nanofibers. The final structures were then dried overnight at RT to eliminate solvent residuals.

Crosslinking of the combined structures. The developed constructs were crosslinked with saturated gluteraldehyde vapor at RT for $48 \mathrm{~h}$. The samples were placed on a metal mesh and put inside a vacuum oven containing an aqueous glutaraldehyde solution $(30 \% \mathrm{v} / \mathrm{v})$. After crosslinking, the constructs were subsequently immersed in glycine solution $(0.02 \mathrm{M})$ for $4 \mathrm{~h}$ to remove unreacted glutaraldehyde. They were then washed several times with distilled water, dried, and stored at dessicator until use. 
Design of thicker scaffolds using a layer-by-layer concept. Although the thickness of the prepared membrane allows to impregnate the microfiber meshes with nanofibers, it would be more difficult to obtain a homogenous structure when the thickness of the fiber meshes increases. To overcome this problem, we propose in this work the use of a layer-by-layer concept to design a thick scaffold with a homogenous nanofiber distribution, even in the interior part of the scaffold. In this method, SPCL fiber mesh membranes with one side deposited with collagen nanofibers were stack together by simply heating at $60^{\circ} \mathrm{C}$, which is the melting point of SPCL. The schematic illustration of this process is presented in Figure 1.

\section{Morphology of nano/micro combined scaffold}

The morphology of the developed structures was observed by a scanning electron microscope (SEM; Leica Cambridge S360) and an optical microscope. The samples were further examined by SEM to evaluate the influence of crosslinking in the fiber morphology and overall structure.

Further and in a way to complement SEM data, the 3D architecture of the collagen nano-network was assessed by confocal laser scanning microscopy (CLSM; Olympus IX81) after staining with antibody against type I collagen. The scaffolds were incubated for $1 \mathrm{~h}$ at RT with the primary antibody mouse anti-bovine (1:100; Sigma-Aldrich). After phosphate-buffered saline (PBS) washing, a second incubation was performed for $1 \mathrm{~h}$ at RT with secondary antibody anti-mouse Alexa Fluor 488 (1:100; Invitrogen). The constructs were washed with PBS, mounted with mounting medium (Vectashield), and observed by CLSM (Olimpus IX81).

\section{Cells, culture conditions, and scaffolds seeding}

A human osteoblast cell line (SaOs-2) was selected to test the developed structures. The cells were cultured in completed medium Dulbecco's modified Eagle's medium low glucose (Sigma-Aldrich) supplemented with 10\% fetal bovine serum (Sigma) and 1\% antibiotics/antimicotics (SigmaAldrich) until they reached the confluence. They were then trypsinized and seeded onto the samples using the density of $2 \times 10^{5}$ cells/scaffold. The cells on the combined structures were allowed to grow for 7 days under standard conditions $\left(37^{\circ} \mathrm{C}, 5 \% \mathrm{CO}_{2}\right)$.

Primary cultures of human endothelial cells (human umbilical vein endothelial cells [HUVECs]) were isolated from the umbilical vein by collagenase digestion according to a previously published method. ${ }^{29}$ HUVECs were cultured in M199 medium (Sigma-Aldrich) supplemented with fetal calf serum (20\%; Gibco), antibiotics/antimicotics (1\%), glutamax I ( $2 \mathrm{mM}$; Gibco), sodium heparin ( $25 \mu \mathrm{g} / \mathrm{mL}$; Sigma-Aldrich), and ECs growth supplement $(25 \mu \mathrm{g} / \mathrm{mL}$; BD Biosciences). Some of the combined structures were precoated with a fibronectin solution (10 $\mu \mathrm{g} / \mathrm{mL}$ PBS; Sigma-Aldrich) for $1 \mathrm{~h}$ at $37^{\circ} \mathrm{C}$. Confluent HUVECs were trypsinized and a suspension of $7.5 \times 10^{4}$ cells was added to each sample. The cell/sample constructs were incubated under standard culture conditions for 3 and 7 days.

\section{Cell imaging}

SEM was the chosen technique for an initial evaluation of the morphology of the cells growing on the developed scaffolds. Samples were fixed with glutaraldehyde in PBS $(2.5 \%)$ for $30 \mathrm{~min}$, dehydrated in increasing concentrations of alcohol, air-dried, and sputter coated with gold before SEM observation (Leica Cambridge S360).

The cellular viability was assessed through the vital dye calcein-AM. Both osteoblast- and HUVEC-seeded combined structures were incubated for $10 \mathrm{~min}$ in the medium supplemented with calcein-AM $(0.1 \mu \mathrm{M})$. This vital dye is internalized by viable cells that by the action of active intracellular esterases convert into a green fluorescent impermeable dye. Then samples were mounted in mounting medium Vectorshield (Vector) and observed by CLSM (Olimpus IX81).

\section{Platelet-endothelial cell adhesion molecule-1 and phalloidin expression}

Samples were fixed with a solution of formalin $(3.7 \%$; Sigma) and permeabilized with Triton ( $0.1 \%)$ for $5 \mathrm{~min}$ at RT. The scaffolds cultured with HUVECs were stained for the cell-cell adhesion molecule (platelet-endothelial cell adhesion molecule-1 [PECAM-1]). For this, EC-seeded scaffolds were incubated for $45 \mathrm{~min}$ at RT with the primary antibody mouse anti-human PECAM-1 (1:50; Dako). A second incubation was performed with the secondary antibody antimouse Alexa Fluor 488 for $45 \mathrm{~min}$ at RT.

Actin fibers of both HUVECs and SaOs cells growing on the scaffolds under analysis were observed by fluorescent phalloidin. Fixed and permeabilized samples were incubated for $20 \mathrm{~min}$ with Alexa Fluor-conjugated phalloidin (1:80; Sigma) at RT.

In both PECAM-1 and phalloidin staining experiments, the nuclei were counterstained with $4^{\prime}$,6-diamidino-2-phenylindole

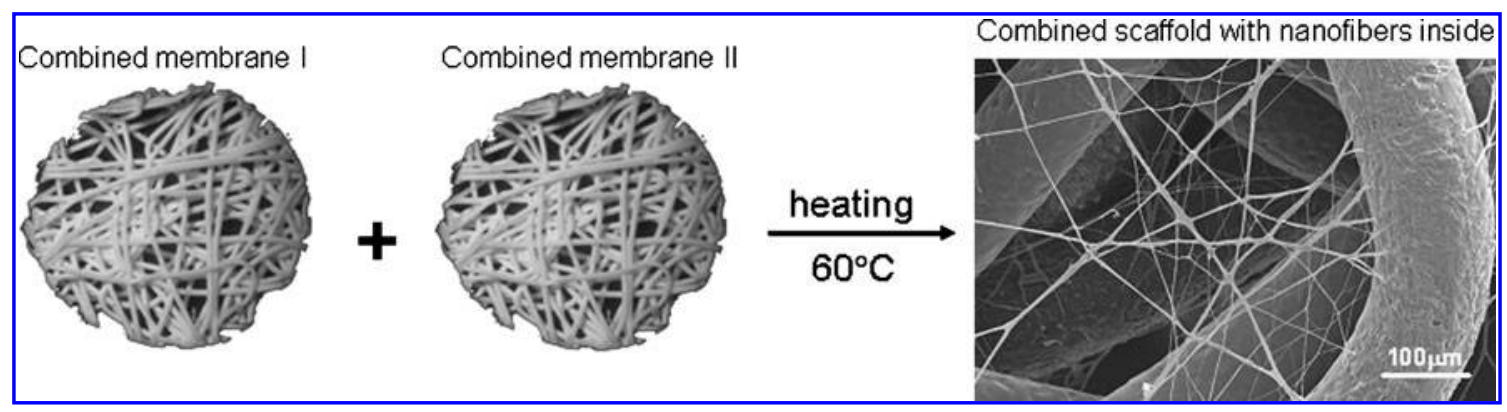

FIG. 1. Schematic illustration of layer-by-layer concept (thickness of the fiber mesh membranes is about $500 \mu \mathrm{m})$. 
(DAPI) (1:1000; Sigma) for $5 \mathrm{~min}$ at RT. To remove the excess of reagents between each step, a washing with PBS was always performed.

\section{Cell proliferation assay}

After 3 and 7 days of culture, cell proliferation was assessed by means of measuring mitochondrial dehydrogenase activity using Cell Titer 96 Aqueous One Solution Cell Proliferation Assay kit (Promega), according to the manufacturer's protocol. This assay is based on the bioreduction of the substrate, (3-(4,5-dimethylthiazol-2-yl)-5(3-carboxymethoxyphenyl)2(4-sulphophenyl)-2H tetrazolium) (MTS), into a brown formazan product by NADPH or NADP produced by dehydrogenase enzyme in metabolically active cells. According to the standard procedure, the triplicates of the samples were washed with sterile PBS and placed in new culture wells. Fresh medium without phenol red and MTS reagent were added to each well in 5/1 ratio. The reaction was carried out by incubating the cell/scaffold constructs with this medium for $3 \mathrm{~h}$ at $37^{\circ} \mathrm{C}$ in a humidified atmosphere containing $5 \%$ of $\mathrm{CO}_{2}$. In the end of the reaction, incubated medium $(100 \mu \mathrm{L})$ was transferred to 96-well plate and optical density was read at $490 \mathrm{~nm}$ in a micro-plate reader (Synergy HT; Bio-tek). The results are expressed as the average absorbance of triplicate samples.

\section{Statistical analysis}

All data related to MTS assay were reported means \pm standard deviation for $n=3$ for each sample. Values were analyzed by using a two-tailed student's $t$-test and $p$-values $<0.05$ were considered significant.

\section{Results}

\section{Morphology of the developed structures}

Optical microscope image demonstrates the structural organization of both nano- and microfiber networks in a combined structure (Fig. 2A). SPCL microfibers with a diameter of $100 \mu \mathrm{m}$ create a nonwoven mesh structure while collagen nanofibers laid onto them with a random orientation. This structural organization can be seen more clearly by SEM in Figure 2B. The average diameter of collagen nanofibers was measured to be around $400 \mathrm{~nm}$. In addition, there was no bead formation on the nanofibers, which indicates that optimum experimental parameters for electrospinning were used for this particular study.

The collagenous nature of the nanofibers in the combined structures was assessed by immunohistochemistry with antibody raised against type I collagen (Fig. 2C). CLSM confirmed the type I collagen nature of the nanofibers and disclosed their spatial distribution on the combined structures. On the combined structures type I collagen nanofibers were found on top of the microfibers and spanning between them. Nanofibers covering SPCL microfibers provided them with a type I collagen coating. Between microfibers, randomly electrospun nanofibers originated a branched network of type I collagen.

To maintain the structural and mechanical integrity, scaffolds made of collagen should be crosslinked. There are several methods that can be used for collagen crosslinking. Herein, we applied chemical crosslinking strategy based on the application of glutaraldehyde vapor. Using glutaraldeyhde in the vapor form would not only allow the
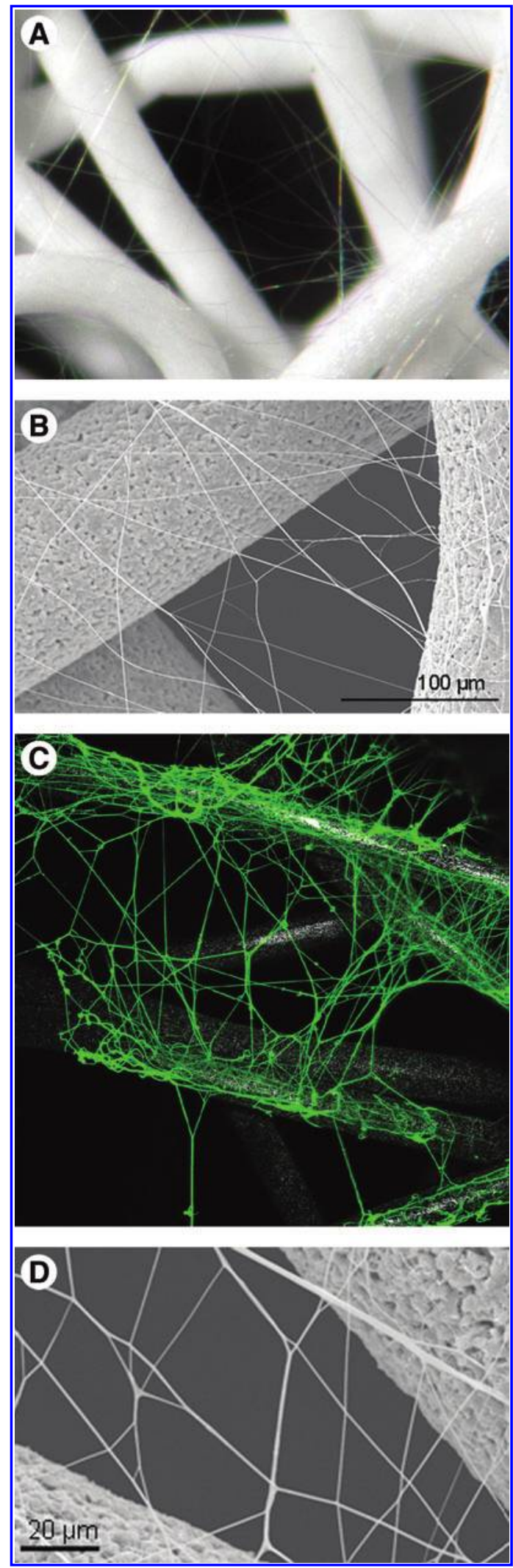

FIG. 2. Structural organization of the combined constructs observed by (A) optical microscopy, 50×, and (B) SEM. (C) Immunostaining with antibody against type I collagen. (D) Morphology of the structures after crosslinking with glutaraldehyde. SEM, scanning electron microscopy. Color images available online at www.liebertonline.com/ten. 

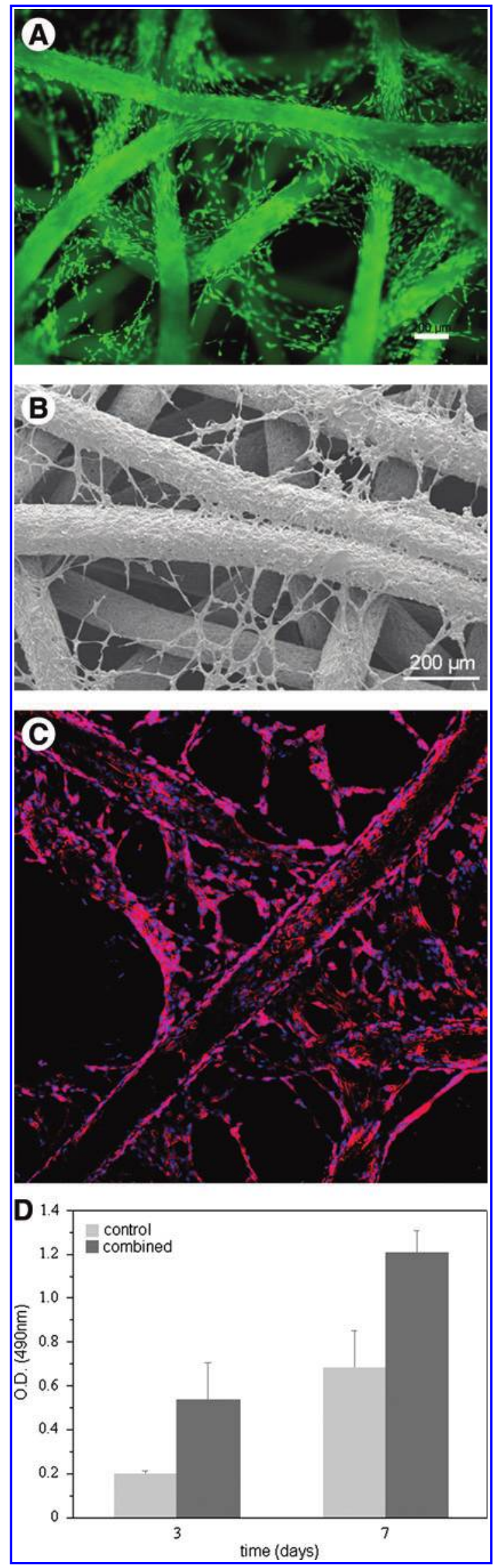

crosslinker to penetrate into the deepest part of the samples, but also will minimize the toxic effect of this reaction. It is also important to note that glycine is used to remove unreacted glutaraldehyde. It has been reported that amino groups of glycine can react easily with the aldehyde groups that are come from unreacted gluteraldeyde. ${ }^{30}$ After crosslinking, it is important to analyze the effect of the reaction on the morphology of the collagen nanofibers, as well as on the integrity of the overall scaffold structure. As it is presented in Figure 2D, crosslinking process had no side effect on the nanofiber morphology and the combined structures retained their structural integrity as before crosslinking.

Figure 1 shows a schematic illustration of thick combined scaffolds prepared by means of using the layer by layer concept. SEM analysis indicated that collagen nanofibers distributed homogenously overall scaffold, including the interior part. Moreover, both micro and nanofiber meshes were able to maintain their original structural organizations in the same construct even after the mild heating process and create a thicker scaffold.

\section{Osteoblast cell attachment and proliferation}

The viability of the osteoblasts seeded on the combined structures was visually determined by calcein-AM staining and by its conversion into a green fluorescent and impermeable product by esterases of viable cells. After 3 days of culture, cells could attach and cover both nano- and microfibers of the combined structures (Fig. 3A). They were able to bridge between SPCL microfibers by using collagen nanofibers. The influence of nanofibers on the cell morphology can be seen better in the SEM image (Fig. 3B). In the presence of nanofibers, osteoblast were stretching themselves along the nanofibers and making bridges between microfibers. This morphological change of the cells led a different cytoskeleton pattern as it was observed by phalloidin staining (Fig. 3C). Cell proliferation was followed by an MTS assay for 7 days (Fig. 3D). Cell proliferation increased form days 3 to 7 for both control (samples without collagen nanofibers) and combined scaffolds. Compared to the cell proliferation in the presence of nanofibers, metabolic activity of the cells was significantly $(p<0.05)$ higher for all time period tested.

\section{EC attachment and metabolic activity}

On what concerns to ECs, cell adhesion was assessed in the absence and presence of fibronectin as a precoating. Currently available polymeric materials do not generally support EC growth without coating with adhesive proteins, such as fibronectin; therefore, this was used as a positive control. After 3 days of culture both noncoated and fibronectincoated combined structures were covered by viable ECs

FIG. 3. Osteoblast-like cells on the combined structures after 3 days of culture. (A) Confocal microscopy of osteoblasts after staining with the vital dye calcein-AM. (B) SEM images of osteoblasts on the developed structures. (C) Phalloidin staining of osteoblasts seeded on combined structures. Nuclei were counterstained with 4',6-diamidino-2-phenylindole (DAPI) and the immunofluorescent micrographs were obtained by confocal microscopy. Original magnification: $\times 100$ (D) Proliferation of osteoblasts was determined by MTS assay. Color images available online at www.liebertonline.com/ten. 

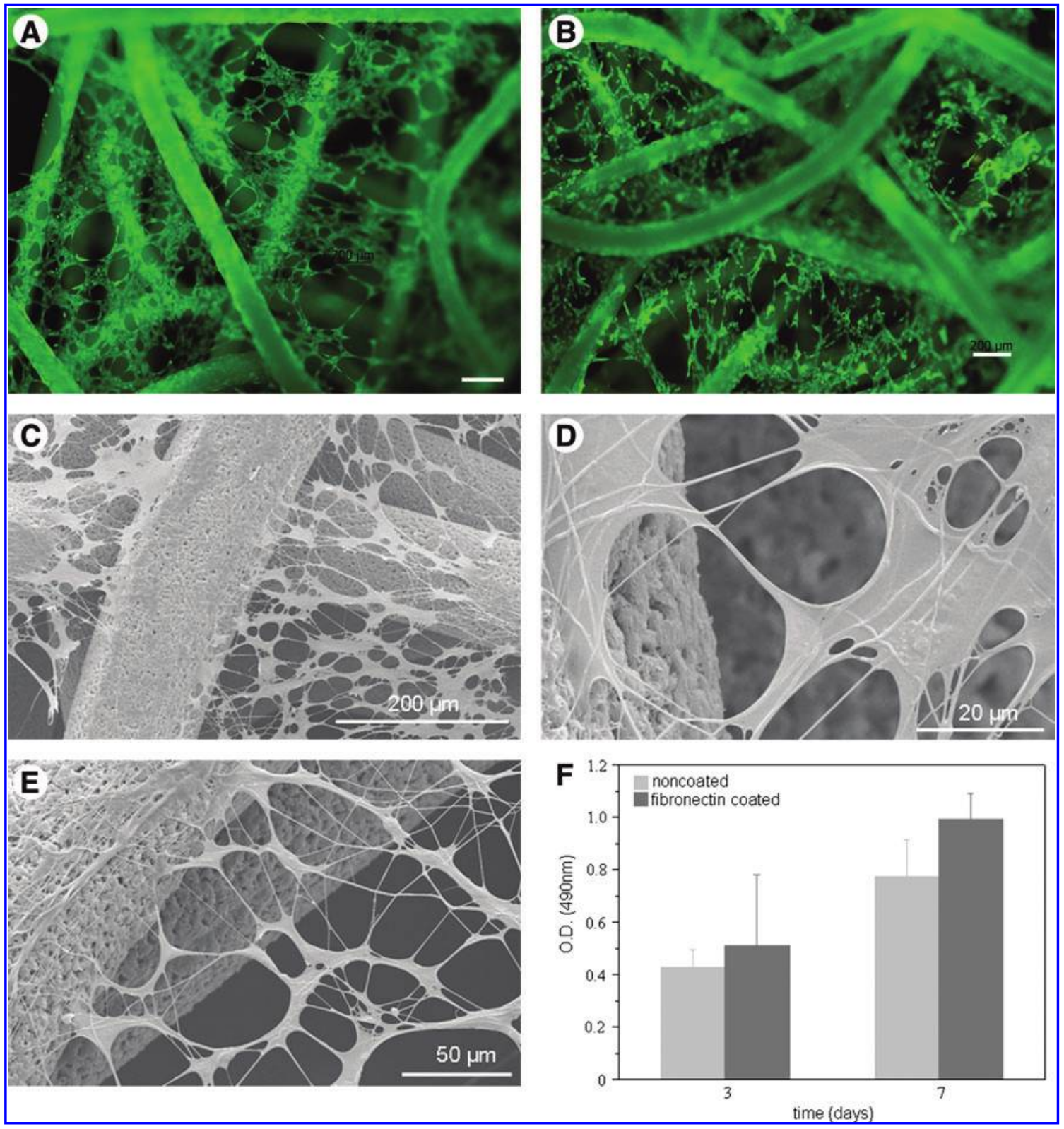

FIG. 4. HUVECs on the combined structures after 3 days of culture and the influence of fibronectin precoating on viability, morphology, and proliferation; (A, C, D) noncoated constructs and (B, E) precoated with fibronectin. Confocal microscopy of HUVECs after staining with the vital dye calcein-AM (A, B). SEM images of HUVECs on the developed structures (C-E). Proliferation of HUVECs was determined by MTS assay (F). Scale bar: (A, B) $200 \mu \mathrm{m}$. HUVECs, human umbilical vein endothelial cells. Color images available online at www.liebertonline.com/ten.

(Fig. 4A, B). SEM data revealed flat and spread ECs on the nano-network and arranged in circular structures (Fig. 4C), an arrangement quite distinct from that observed for the osteoblasts. A higher magnification unveiled not only that ECs are growing on top of type I collagen nanofibers but also that these nanofibers are being integrated into the cellular cytoplasma (Fig. 4D). When analyzing the effect of precoating the scaffold with fibronectin, it was observed the same cell adhesion pattern and cell morphology in the absence of coating. MTS data further support these finding insofar as after 3 and 7 days there was not observed any significant difference in the of ECs growing on fibronectin-coated and noncoated combined structures (Fig. 4E).
Cell morphology of ECs growing on noncoated and fibronectin-coated combined structures was further unveiled after actin cytoskeleton staining with phalloidin. On the nano-network ECs' cytoskeleton followed the alignment dictated by type I collagen nanofibers (Fig. 5A). One should particularly note that there are single cells growing on the individual nanofibers with their actin filaments directed in a unidirectional way. When looking at the positive control, scaffolds precoated with fibronectin, no difference was observed in the pattern of cytoskeleton in relation to nano-/ microfiber combined scaffold without coating (Fig. 5B).

Expression of the cell junction PECAM-1, the major hallmark of the endothelia, was assessed by immunohisto- 

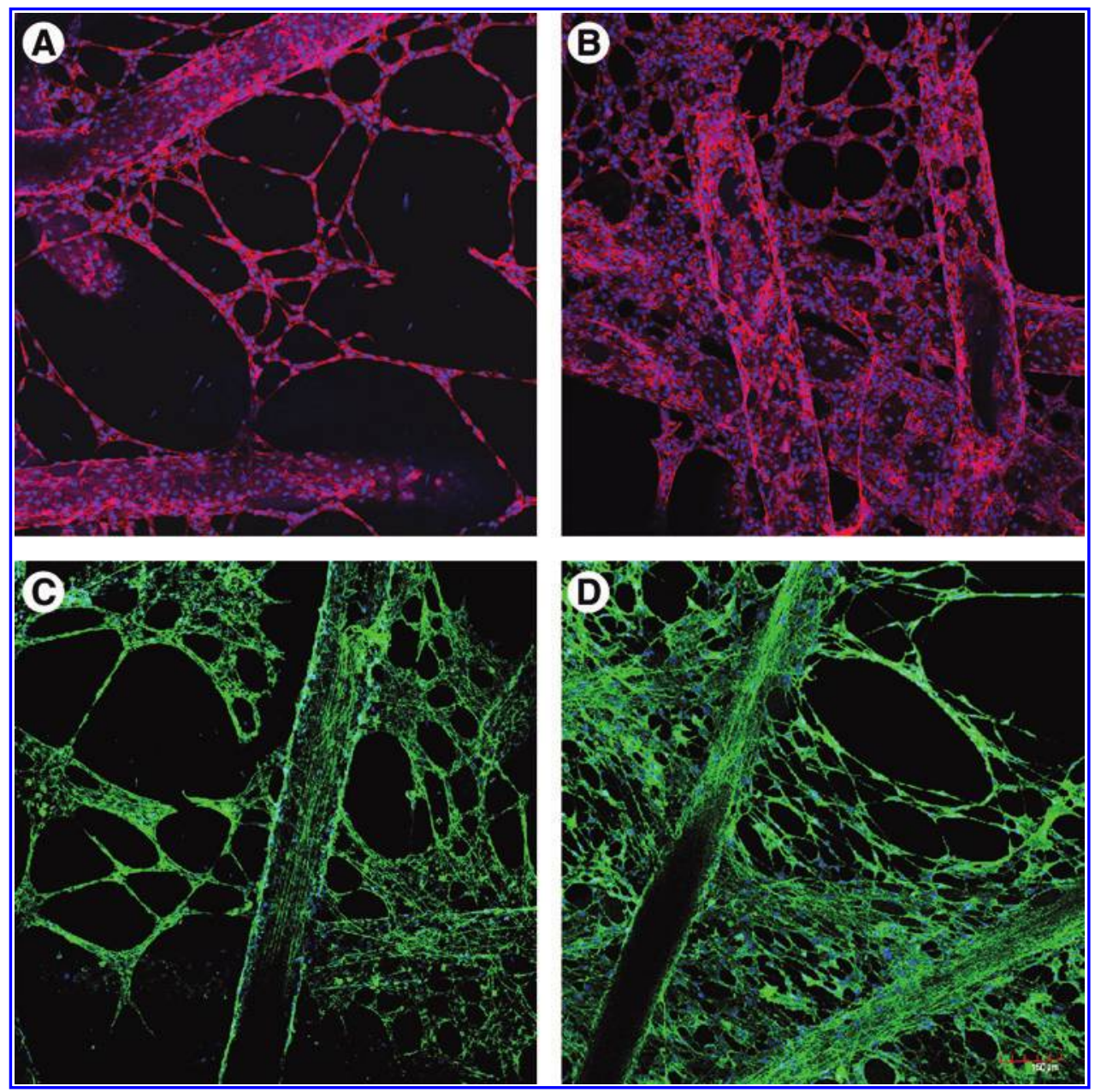

FIG. 5. Phalloidin (A, B) and platelet-endothelial cell adhesion molecule-1 staining (C, D) of HUVECs seeded on combined structures noncoated (A, C) and fibronectin coated (B, D). Nuclei were counterstained with DAPI and the immunofluorescent micrographs were obtained by confocal microscopy. Original magnification: $\times 100$. Color images available online at www.liebertonline.com/ten.

chemistry and observed by CLSM. ECs growing on the combined structures were able to express PECAM-1 at cellcell borders (Fig. 5C). Expression of this important molecule was observed both on micro- and nanofibers. Once more, fibronectin coating was not required for the endothelial phenotype of ECs on the developed combined structures and the same PECAM-1 staining between adjacent cells was observed in the two conditions under study (Fig. 5C, D).

\section{Discussion}

Wet-spun microfiber meshes made of SPCL polymeric blend and electrospun nanofibers from collagen were joined into the same construct, giving rise to combined structures. For that, a two-step methodology was used, a concept and technology previously developed and reported by Tuzlakoglu et al. ${ }^{31,32}$ This new construct is proposed as a manmade equivalent of ECM, which could mimic the physical and chemical structure of it. SPCL microfiber meshes constitute the macro support for the cells, whereas collagen nanofibers laid on them aim to mimic the collagen fibrils of natural ECM. The role of long and stiff collagen fibrils in ECM is to serve structural support as well as connecting and recognition function between cells and the matrix. ${ }^{10}$ Collagen has maintained a highly conserved aminoacids sequence that provides binding sites for integrins presented in cell mem- branes. In fact, integrin binding is a key factor for the necessary cell-ECM communication that is lacking when synthetic polymers are used. ${ }^{33}$ Besides the above-mentioned importance, the choice of collagen relays in the fact that is the most abundant protein within the natural ECM.

To evaluate the structural similarity of the developed combined structures to natural ECM, we used optical and scanning electron microscopy observation. Further, confocal microscopy was selected to assess and observe collagen nanofibers, which were supposed to play a role in chemically mimicking besides the structural one. Structures prepared from micro- and nanofibers demonstrated an architecture with randomly distributed nanofibers that matches the one in the natural ECM. ${ }^{10}$ In electrospinning experiments, processing conditions, particularly processing time, were optimized to create nanofibers in appropriate deposition amount and size. The optimum used conditions were resulted in a random deposition of nanofibers on the microfiber meshes. The average diameter of nanofibers was around $400 \mathrm{~nm}$, which coincides well with the collagen fiber bundle diameter characteristic of the natural ECM. ${ }^{34}$ Immunohistochemistry results confirmed the collagenous nature of nanofibers that were deposited on SPCL microfiber meshes.

The present article introduces the layer-by-layer concept, a novel approach that we have developed to be able to design a structure resembling natural ECM, but that simultaneously 
takes into account the needs for real clinical applications. This rather simple method allowed us to create a construct with a requested thickness for implantation, by means of combining several microfiber meshes with homogenously distributed nanofibers in a single scaffolding material.

The structural merits of developed constructs were evaluated by assessing cellular responses of two important cell types in bone repair (osteoblasts and endothelial) and their viability, cytoskeleton, and expression markers. Data published in this study clearly demonstrated that osteoblast-like cells cultured on the combined structures were able to stretch themselves along the nanofibers, while maintaining their typical spindle-like morphology on the microfibers. The surface of SPCL microfibers was also covered by collagen nanofibers as it was observed by SEM analysis. With respect to this indication, we can claim that the morphological changes of osteoblasts were influenced not only by the chemical nature of the material but also by its the structural organization.

The effect of nano-/microfiber combination on cell viability and metabolic activity was screened using an MTS assay. The presence of collagen nanofibers in the structure resulted in an increase of metabolic activity and growth rate when directly compared to a scaffold without nanofibers. Similar results were previously reported with SaOs-2 and rat bone marrow stromal cells in culture on nano- and micro-combined structure made of SPCL. ${ }^{31}$ Previous reports have demonstrated that type I collagen enhances bone cell viability and growth. ${ }^{35,36}$ It has been used to coat metallic implant to enhance osteoblast spreading that results in a more rapid formation of focal adhesions and their associated stress fibers. ${ }^{37,38}$ Our results suggest that the presence of type I collagen nanofibers appears to influence the cell viability of the osteoblast-like cells cultured on the developed structures. Besides the chemical influence of collagen, the previously deposited nanofibers reduce large void spaces between microfibers and create larger surface area that the cells can adhere from the very beginning. With respect to this phenomenon, the presence of collagen nanofibers in the constructs initially clearly increases the cell seeding efficiency and later on results in a higher cellular metabolic activity.

Cellular adhesion, spreading, and migration is known to be dependent on its cytoskeleton system, including actin filaments. ${ }^{39}$ The cytoskeletal organization of the cells also ordinates the morphological organization of ECM. Therefore, we performed fluorescent phalloidin staining to observe actin filaments in the cytoskeleton of the cells. A clear difference in actin filaments was observed between the cells growing on the microfibers and the nanofibers. Due to the effect of collagen nanofibers, cytoskeleton of the cells growing on nanofibers showed more elongated shape than the one growing on microfibers. Since the cell shape is modulated by polymerization of actin filaments, these results can explain the morphological changes of osteoblast growing on nanofibers, which was observed by SEM.

Presently, one of the major hurdles in the clinical application of tissue engineering to repair metabolically demanding tissues (e.g., bone) is the absence of a capillary bed linking the construct to the host blood system. ${ }^{40}$ Due to their active role in angiogenesis, ECs are a key cell type. ${ }^{41}$ Besides that, ECs are pivotal members of a complex interactive communication network in bone. ${ }^{42}$ Therefore, the chemical composition as well as architecture of combined structures was designed envisioning not only bone-forming cells but also ECs. Type I collagen, together with SPCL, is one of the building blocks of this scaffold and is the major constituent of the extracellular matrices to which proliferating ECs are exposed in injured tissue. ${ }^{43}$ Moreover, collagen also provides adhesive support for osteoblasts, as it has been discussed above. $^{44}$ Therefore, one of the objectives of including a nanonetwork of type I collagen was not only to supply a nanorange physical support for cells but also to provide a cell adhesion promoter. This last aspect is especially important for ECs once they are very demanding and dependent in terms of substrate adhesion. Normally, a very common procedure to improve EC adhesion to the substrate is a precoating with molecules from ECM such as fibronectin. ${ }^{45-47}$ In this work we evaluated the ability of the combined structures to support the growth of ECs without the requirement of any additional precoating. As a positive control the scaffolds were precoated with fibronectin. ECs adhered to uncoated combined structures, remained viable, and exhibited a flat and stretch morphology. The same cell adhesion pattern and cellular morphology were observed for combined structures with fibronectin coating. Metabolic activity quantification of ECs further supported the fact that no significant difference was observed between the positive control and uncoated combined structures. These overall results indicate that precoating with fibronectin did not further improve cell adhesion, viability, or influenced cell morphology.

Angiogenesis is a complex phenomenon with multiple progressive steps toward the end point of new blood vessels formation. It starts with cell adhesion to the new substratum, passing by migration, proliferation, organization in tube-like structures, and deposition of new basement membrane; all these steps have as a common denominator type I collagen. ${ }^{44,48}$ On the nano-network of the developed constructs ECs organized into circular structures resembling the microcapillary-like structures formed during angiogenesis. Also, of particular interest is the intimate contact that was established between ECs and collagen nanofibers. As observed by SEM, nanofibers were integrated within cellular cytoplasma. It has long been recognized that $3 \mathrm{D}$ interstitial collagen type I provokes ECs in culture to undergo marked shape changes that closely imitate the cord-like structures observed during adult angiogenesis. This behavior is EC specific, and this may explain why osteoblasts did not exhibit the same morphology and 3D arrangement on the combined structures.

Regarding ECs' cytoskeleton, phalloidin staining revealed different patterns on the combined structures. On nanofibers the cells were more stretched and with actin filaments aligned in an un-directional way, in contrast to microfibers where cells exhibit a more disperse conformation of actin fibrils. These differences reflex the distinct biochemical and physiochemical natures of the substrata on the combined structures, which ultimately will dictate diverse cell functions such as migration, proliferation, among others. ${ }^{10}$

ECs' migration is an important factor for angiogenesis, particularly during sprouting of new blood vessels from the existing vasculature. ${ }^{44}$ The inclusion of the nano-network was also designed to increment ECs' motility. This was 
based on data indicating that ECs are most motile in sparse culture in which they establish few contacts with their neighbors, in opposition to cells incorporated into a confluent monolayer that reveal reduced movements. ${ }^{49,50}$ Therefore, it is expected that when exposed to an angiogenic environment, ECs on the different fiber size of the combined structure will behave differently; sparse ECs on nanofibers will be more motile than confluent cells lying down on microfibers. Also, the collagenous nature of the nano-network will probably contribute to this motility. This assumption is in keeping with in vitro studies that have shown that type I collagen not only supports chemotactic migration of ECs but is also responsible for haptotactic migration. ${ }^{44}$

For vessel formation, networking and remodeling cell-cell adhesion are particularly important. ${ }^{50}$ PECAM-1 is a cell adhesion molecule, concentrated at the lateral junctions of adjacent ECs and is a major hallmark of the endothelium. On the combined structures, ECs contacted with their neighbor cells and expressed PECAM-1 at the borders. PECAM-1 staining was present on the overall structure, indicating that the effect of micro- and nanometric fiber size did not affect cell-cell communication. These findings confirm the normal endothelial phenotype, being also a good indicator of the interactions between ECs and the novel combined structures.

\section{Conclusions}

We developed combined structures as a new construct nature-inspired that recreates the physical and chemical environment of bone matrix. These structures were obtained by a two-step methodology where nanofibers of type I collagen, with an average size of $400 \mathrm{~nm}$, were electrospun on the macro support made from SPCL fiber mesh. The collagenous nature of the nano-network was confirmed by immunhistochemistry and its 3D architecture characterized by several microscopy techniques. Further, it was proved the efficacy of the layer-by-layer concept as an approach to create thicker scaffolds.

Regarding cellular interactions, combined structures were able to support the adhesion and growth of both osteoblasts and ECs. About osteoblasts, the presence of type I collagen nanofibers increased metabolic activity and the surface area available for cell spanning. In the particular case of ECs, the inclusion of type I collagen obviated the need of precoating with fibronectin and cells organized into circular structures resembling angiogenic organization.

Our findings indicate that combined structures are an appropriate human equivalent of natural ECM for bone tissue engineering.

\section{Acknowledgments}

K. Tuzlakoglu and M.I. Santos thank the Portuguese Foundation for Science and Technology for their Ph.D. scholarship (SFRH/BD/8502/2002 and SFRH/BD/13428/ 2003). This work was partially supported by FCT Foundation for Science and Technology, through funds from the POCTI and/or FEDER programs and by the European Union funded STREP Project HIPPOCRATES (NMP3-CT-2003-505758). This work was carried out under the scope of the European NoE EXPERTISSUES (NMP3-CT-2004-500283). Work developed under the cooperation agreement between UM-3B's research group and the Hospital de S. Marcos, Braga. The authors thank to L. Goreti Pinto for her help on confocal microscopy studies.

\section{Disclosure Statement}

No competing financial interests exist.

\section{References}

1. Warren, S.M., Steinbrech, D.S., Mehrara, B.J., Saadeh, P.B., Greenwald, J.A., Spector, J.A., Bouletreau, P.J., and Longaker, M.T. Hypoxia regulates osteoblast gene expression. I Surg Res 99, 147, 2001.

2. Unger, R.E., Wolf, M., Peters, K., Motta, A., Migliaresi, C., and James Kirkpatrick, C. Growth of human cells on a nonwoven silk fibroin net: a potential for use in tissue engineering. Biomaterials 25, 1069, 2004.

3. Seol, Y.J., Lee, J.Y., Park, Y.J., Lee, Y.M., Young-Ku, Rhyu, I.C., Lee, S.J., Han, S.B., and Chung, C.P. Chitosan sponges as tissue engineering scaffolds for bone formation. Biotechnol Lett 26, 1037, 2004.

4. Tuzlakoglu, K., Pashkuleva, I., Rodrigues, M.R., Gomes, V.L., Müller, R., and Reis, R.L. A new route to produce starch-based fiber mesh scaffolds by wet spinning and the improvement in cell attachment and proliferation by tailoring their surface properties. J Biomed Mater Res Part A 92, 369, 2009.

5. Sombatmankhong, K., Sanchavanakit, N., Pavasant, P., and Supaphol, P. Bone scaffolds from electrospun fiber mats of poly (3-hydroxybutyrate), poly(3-hydroxybutyrate-co-3hydroxyvalerate) and their blend. Polymer 48, 1419, 2007.

6. Gugala, Z., and Gogolewski, S. The in vitro growth and activity of sheep osteoblasts on three-dimensional scaffolds from poly(L/DL-lactide) 80/20\%. J Biomed Mater Res Part A 75A, 702, 2005.

7. Yoon, C.H., Hur, J., Park, K.W., Kim, J.H., Lee, C.S., Oh, I.Y., Kim, T.Y., Cho, H.J., Kang, H.J., Chae, I.H., Yang, H.K., Oh, B.H., Park, Y.B., and Kim, H.S. Synergistic neovascularization by mixed transplantation of early endothelial progenitor cells and late outgrowth endothelial cells: the role of angiogenic cytokines and matrix metalloproteinases. Circulation 112, 1618, 2005.

8. Li, T., Yu, Y.T., Wang, J., and Tang, T.S. 1,25-Dihydroxyvitamin $\mathrm{D}(3)$ stimulates bone neovascularization by enhancing the interactions of osteoblasts-like cells and endothelial cells. J Biomed Mater Res A 86, 583, 2008.

9. Harkness, R.D. Biological functions of collagen. Biol Rev Camb Philos Soc 36, 399, 1961.

10. Lutolf, M.P., and Hubbell, J.A. Synthetic biomaterials as instructive extracellular microenvironments for morphogenesis in tissue engineering. Nat Biotechnol 23, 47, 2005.

11. Huang, Z.M., Zhang, Y.Z., Kotaki, M., and Ramakrishna, S. A review on polymer nanofibers by electrospinning and their applications in nanocomposites. Composites Sci Technol 63, 2223, 2003.

12. Heng, B.C., Cao, T., Stanton, L.W., Robson, P., and Olsen, B. Strategies for directing the differentiation of stem cells into the osteogenic lineage in vitro. J Bone Miner Res 19, 1379, 2004.

13. Laroche, M. Intraosseous circulation from physiology to disease. Joint Bone Spine 69, 262, 2002.

14. Garcia, A.J., and Reyes, C.D. Bio-adhesive surfaces to promote osteoblast differentiation and bone formation. I Dent Res 84, 407, 2005.

15. Ignatius, A., Blessing, H., Liedert, A., Schmidt, C., Neidlinger-Wilke, C., Kaspar, D., Friemert, B., and Claes, L. Tissue 
engineering of bone: effects of mechanical strain on osteoblastic cells in type I collagen matrices. Biomaterials 26, 311, 2005.

16. Deroanne, C.F., Lapiere, C.M., and Nusgens, B.V. In vitro tubulogenesis of endothelial cells by relaxation of the coupling extracellular matrix-cytoskeleton. Cardiovasc Res 49, 647, 2001.

17. Palmieri, D., Camardella, L., Ulivi, V., Guasco, G., and Manduca, P. Trimer carboxyl propeptide of collagen I produced by mature osteoblasts is chemotactic for endothelial cells. J Biol Chem 275, 32658, 2000.

18. Wenger, A., Stahl, A., Weber, H., Finkenzeller, G., Augustin, H.G., Stark, G.B., and Kneser, U. Modulation of in vitro angiogenesis in a three-dimensional spheroidal coculture model for bone tissue engineering. Tissue Eng 10, 1536, 2004.

19. Muschler, G.F., Nakamoto, C., and Griffith, L.G. Engineering principles of clinical cell-based tissue engineering. J Bone Joint Surg Am 86-A, 1541, 2004.

20. Rouwkema, J., De Boer, J., and Van Blitterswijk, C.A. Endothelial cells assemble into a 3-dimensional prevascular network in a bone tissue engineering construct. Tissue Eng 12, 2685, 2006.

21. Stahl, A., Wenger, A., Weber, H., Stark, G.B., Augustin, H.G., and Finkenzeller, G. Bi-directional cell contactdependent regulation of gene expression between endothelial cells and osteoblasts in a three-dimensional spheroidal coculture model. Biochem Biophys Res Commun 322, 684, 2004.

22. Levenberg, S., Rouwkema, J., Macdonald, M., Garfein, E.S., Kohane, D.S., Darland, D.C., Marini, R., van Blitterswijk, C.A., Mulligan, R.C., D'Amore, P.A., and Langer, R. Engineering vascularized skeletal muscle tissue. Nat Biotechnol 23, 879, 2005.

23. Choong, C.S., Hutmacher, D.W., and Triffitt, J.T. Co-culture of bone marrow fibroblasts and endothelial cells on modified polycaprolactone substrates for enhanced potentials in bone tissue engineering. Tissue Eng 12, 2521, 2006.

24. Cassell, O.C., Hofer, S.O., Morrison, W.A., and Knight, K.R. Vascularisation of tissue-engineered grafts: the regulation of angiogenesis in reconstructive surgery and in disease states. Br J Plast Surg 55, 603, 2002.

25. Soker, S., Machado, M., and Atala, A. Systems for therapeutic angiogenesis in tissue engineering. World J Urol 18, 10, 2000.

26. Kim, B.S., and Mooney, D.J. Development of biocompatible synthetic extracellular matrices for tissue engineering. Trends Biotechnol 16, 224, 1998.

27. Gomes, M.E., Godinho, J.S., Tchalamov, D., Cunha, A.M., and Reis, R.L. Alternative tissue engineering scaffolds based on starch: processing methodologies, morphology, degradation and mechanical properties. Mat Sci Eng C Biomimetic Supramol Syst 20, 19, 2002.

28. Freshney, R.I. Culture of Animal Cells: A Manual of Basic Technique. New York: John Wiley \& Sons, Inc., 1993.

29. Jaffe, E.A., Nachman, R.L., Becker, C.G., and Minick, C.R. Culture of human endothelial cells derived from umbilical veins. Identification by morphologic and immunologic criteria. J Clin Invest 52, 2745, 1973.

30. Yamaguchi, A., Ishizuya, T., Kintou, N., Wada, Y., Katagiri, T., Wozney, J.M., Rosen, V., and Yoshiki, S. Effects of BMP-2, BMP-4, and BMP-6 on osteoblastic differentiation of bone marrow-derived stromal cell lines, ST2 and MC3T3-G2/ PA6. Biochem Biophys Res Commun 220, 366, 1996.
31. Tuzlakoglu, K., Bolgen, N., Salgado, A.J., Gomes, M.E., Piskin, E., and Reis, R.L. Nano- and micro-fiber combined scaffolds: a new architecture for bone tissue engineering. J Mater Sci Mater Med 16, 1099, 2005.

32. Santos, M.I., Tuzlakoglu, K., Fuchs, S., Gomes, M.E., Peters, K., Unger, R.E., Piskin, E., Reis, R.L., and Kirkpatrick, C.J. Endothelial cell colonization and angiogenic potential of combined nano- and micro-fibrous scaffolds for bone tissue engineering. Biomaterials 29, 4306, 2008.

33. Bowlin, G.L. A new spin on scaffold. Mater Today 7, 64, 2004.

34. Sell, S., Barnes, C., Smith, M., McClure, M., Madurantakam, P., Grant, J., McManus, M., and Bowlin, G. Extracellular matrix regenerated: tissue engineering via electrospun biomimetic nanofibers. Polym Int 56, 1349, 2007.

35. Tesema, Y., Raghavan, D., and Stubbs, J. Bone cell viability on collagen immobilized poly (3-hydroxybutrate-co-3hydroxyvalerate) membrane: effect of surface chemistry. I Appl Polym Sci 93, 2445, 2004.

36. Morra, M., Cassinelli, C., Cascardo, G., Cahalan, P., Cahalan, L., Fini, M., and Giardino, R. Surface engineering of titanium by collagen immobilization. Surface characterization and in vitro and in vivo studies. Biomaterials 24, 4639, 2003.

37. Geissler, U., Hempel, U., Wolf, C., Scharnweber, D., Worch, H., and Wenzel, K.W. Collagen type I-coating of Ti6Al4V promotes adhesion of osteoblasts. I Biomed Mater Res 51, $752,2000$.

38. Roehlecke, C., Witt, M., Kasper, M., Schulze, E., Wolf, C., Hofer, A., and Funk, R.H.W. Synergistic effect of titanium alloy and collagen type I on cell adhesion, proliferation and differentiation of osteoblast-like cells. Cells Tissues Organs 168, 178, 2001.

39. Yushkevich, P.A., Piven, J., Hazlett, H.C., Smith, R.G., Ho, S., Gee, J.C., and Gerig, G. User-guided 3D active contour segmentation of anatomical structures: significantly improved efficiency and reliability. Neuroimage 31, 1116, 2006.

40. Kannan, R.Y., Salacinski, H.J., Sales, K., Butler, P., and Seifalian, A.M. The roles of tissue engineering and vascularisation in the development of micro-vascular networks: a review. Biomaterials 26, 1857, 2005.

41. Ucuzian, A.A., and Greisler, H.P. In vitro models of angiogenesis. World J Surg 31, 654, 2007.

42. Guillotin, B., Bourget, C., Remy-Zolgadri, M., Bareille, R., Fernandez, P., Conrad, V., and Amedee-Vilamitjana, J. Human primary endothelial cells stimulate human osteoprogenitor cell differentiation. Cell Physiol Biochem 14, 325, 2004.

43. Seandel, M., Noack-Kunnmann, K., Zhu, D., Aimes, R.T., and Quigley, J.P. Growth factor-induced angiogenesis in vivo requires specific cleavage of fibrillar type I collagen. Blood 97, 2323, 2001.

44. Davis, G.E., and Senger, D.R. Endothelial extracellular matrix: biosynthesis, remodeling, and functions during vascular morphogenesis and neovessel stabilization. Circ Res 97, 1093, 2005.

45. Tajima, S., Chu, J.S., Li, S., and Komvopoulos, K. Differential regulation of endothelial cell adhesion, spreading, and cytoskeleton on low-density polyethylene by nanotopography and surface chemistry modification induced by argon plasma treatment. J Biomed Mater Res A 84, $828,2008$.

46. Santos, M.I., Fuchs, S., Gomes, M.E., Unger, R.E., Reis, R.L., and Kirkpatrick, C.J. Response of micro- and macrovascular 
endothelial cells to starch-based fiber meshes for bone tissue engineering. Biomaterials 28, 240, 2007.

47. Unger, R.E., Peters, K., Wolf, M., Motta, A., Migliaresi, C., and Kirkpatrick, C.J. Endothelialization of a non-woven silk fibroin net for use in tissue engineering: growth and gene regulation of human endothelial cells. Biomaterials 25, 5137, 2004.

48. Breithaupt-Faloppa, A.C., Kleinheinz, J., and Crivello, O., Jr. Endothelial cell reaction on a biological material. J Biomed Mater Res B Appl Biomater 76, 49, 2006.

49. Osborn, E.A., Rabodzey, A., Dewey, C.F., Jr., and Hartwig, J.H. Endothelial actin cytoskeleton remodeling during mechanostimulation with fluid shear stress. Am J Physiol Cell Physiol 290, C444, 2006.

50. Liebner, S., Cavallaro, U., and Dejana, E. The multiple languages of endothelial cell-to-cell communication. Arterioscler Thromb Vasc Biol 26, 1431, 2006.
Address correspondence to: Kadriye Tuzlakoglu, B.Eng., M.Sc., Ph.D. 3B's Research Group-Biomaterials, Biodegradables and Biomimetics

Headquarters of the European Institute of Excellence on Tissue Engineering and Regenerative Medicine University of Minho AvePark 4806-909 Guimarães Portugal

E-mail: kadriye@dep.uminho.pt

Received: March 23, 2010

Accepted: September 7, 2010

Online Publication Date: October 27, 2010 



\section{This article has been cited by:}

1. Rebecca L. Dahlin , F. Kurtis Kasper, Antonios G. Mikos . 2011. Polymeric Nanofibers in Tissue Engineering. Tissue Engineering Part B: Reviews 17:5, 349-364. [Abstract] [Full Text] [PDF] [PDF Plus]

2. Chongyun Bao, Wenchuan Chen, Michael D. Weir, Wahwah Thein-Han, Hockin H.K. Xu. 2011. Effects of electrospun submicron fibers in calcium phosphate cement scaffold on mechanical properties and osteogenic differentiation of umbilical cord stem cells. Acta Biomaterialia . [CrossRef]

3. Jong Kyu Hong , Sundararajan V. Madihally . 2011. Next Generation of Electrosprayed Fibers for Tissue Regeneration. Tissue Engineering Part B: Reviews 17:2, 125-142. [Abstract] [Full Text] [PDF] [PDF Plus] 